\title{
Cognitive training through DVD: Working memory task as a tool for rehabilitation in elders
}
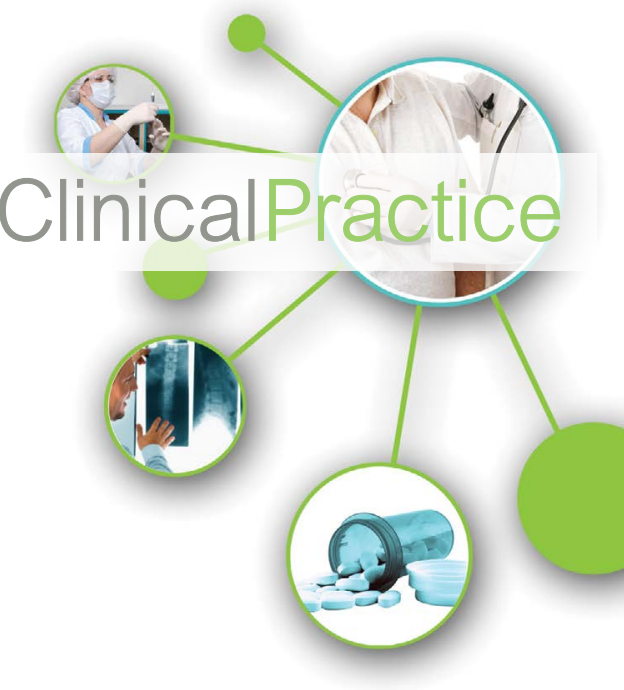

\begin{abstract}
Studies have shown that working memory tasks minimize the cognitive disabilities associated with old age. In this context, the present study examined the bavioral changes of working memory in elders during a cognitive training through DVDs. The results show that there significance in MMSE tests $(p=0.001)$, self-evaluation $(p=0.002)$ and verbal fluency test in the semantic category $(p=0.022)$. However, verbal fluency tests of spelling criterion and free evocation did not present significant differences $(p \geq 0.05)$. Based on the findings, we suggest that recurring training with working memory tasks through DVDs promotes neural adaptations compared to retaining and recalling the memory, promoting a compensatory effect in the performance and maintenance of cognitive skills. In addition, the training of working memory through DVDs offers a non-invasive, non-pharmacological and low-cost alternative for rehabilitation.
\end{abstract}

\section{Keywords: Cognitive training, working memory, elders, rehabilitation.}

\section{Introduction}

The world population ageing is a phenomenon that has occurred in recent decades, becoming an adjunct in risk of diseases that impair cognition and executive functions [1,2]. In this context, studies that associate training and cognitive rehabilitation attempt to slow down or even minimize the deficiencies from old age, as well as the decline of the neurobiological aspects such memory and executive functions $[3,4]$. Thus, with the findings regarding brain functioning in function of cognitive demands, this, through neuroimaging and electrophysiological mapping, allowed creating effective strategies to enhance the implementation of mechanisms involved in neural memory, and even stop or reverse the cognitive decline caused by old age and lack of intellectual activity [1,2-6].

Memory decline is not only associated with ageing, but mainly with reduced intellective activity [4]. Neural integration machinery, in particular structures such as the prefrontal cortex associated with the hippocampus, is relevant to initial storage, capacity to acquire, retain and recall information from memory, essential for all bodies' survival [7-9]. Based on the analysis of memory-relevant information processing, the main association route in the short to long- term conversion is evidenced by the working memory, based on the attention control model, visuo-spatial processing, in addition to episodic integration for long-term memory [10,11].

Rehabilitation-besed behavioral investigations are likely to determine phenotypes associated with working memory demand [12]. The literature seeks to trace a profile of cognitive training through multimedia that promotes differential sensitivity to cognition stimuli, in order to improve the quality of life of patients with cognitive deficits and healthy individuals with advanced age, maximizing the use of brain functions involved in that neurobiological aspect, with education of compensatory strategies and acquisition of new skills $[2,4,13]$. Cognitive training through multimedia provides the patient's awareness regarding their remaining capacities, in addition to producing compensatory effects on cognitive function $[9,10,14]$. The present study focuses on the training of working memory, since it can reduce the signs and symptoms of cognitive impairment through experience and task recurrence [15]. The proposed training encodes changes in neuronal interconnections and directs them to a mechanism of neural integration, which possibly improves memory skills in elders
Viviane Marques ${ }^{1,2 *}$, Luise Alexandre Rocha Soutinho ${ }^{3}$, Mônica Marins ${ }^{4}$. Victor Marinho ${ }^{5,6}$, Silmar Teixeira ${ }^{5,6}$, Victor Hugo Bastos ${ }^{7}$, Marco Orsini ${ }^{8}$

${ }^{1}$ Master's program in HIV / AIDS and viral hepatitis, University of Rio de JaneiroUNIRIO, Rio de Janeiro, Brazil

${ }^{2}$ Doctoral Program in Psychoanalysis, Health and Society, Veiga de Almeida University, Rio de Janeiro, Brazil ${ }^{3}$ Multiprofessional Residency Program, University of Rio de Janeiro, Rio de Janeiro, Brazil

${ }^{4}$ Federal University of Rio de Janeiro, ACESIN Laboratory, Rio de Janeiro, Brazil. ${ }^{5}$ Neuro-innovation Technology \& Brain Mapping Laboratory, Federal University of Piauí, Parnaiba, Brazil

${ }^{6}$ The Northeast Biotechnology Network, Federal University of Piaui, Teresina, Brazil

${ }^{7}$ Biotecnologic Program

(PPGCBM), Federal University of Piaui, Parnaíba, Brazil

${ }^{8}$ Master's Program in Applied Science to Health - University of Vassouras and Master's in Local Development Unisuam, Rio de Janeiro, Brazil

${ }^{*}$ Author for correspondence:

vivianemarques@uva.br 
[1,16-18]. Studies show that the memory skills in elders who do not have dementia can improve with instruction and practice, through systematic training $[19,20]$. Therefore, our study presents a biologically plausible framework for improving and maintaining memory-related cognitive aspects, aiming at the elaboration of a non-invasive and easily applicable instrument for working memory training in a geriatric population, based on audio-visual resource and DVD video.

\section{Material and Methods}

\section{- Participants}

We developed a prospective and qualiquantitative study at the Gafrée Guinle University Hospital (HUGG) along the UNIRIO multiprofessional health residency, aiming to test the effects of working memory training through DVD in elders that attend the Grupo Renascer (Reborn Group) - HUGG (TABLE 1). We selected healthy individuals attending the Projeto Renascer (Reborn Project) at the HUGG, both sexes, aged 60 years or more, able to answer the self-evaluation questionnaire and perform the cognitive assessment, in addition to attending at least $75 \%$ of the cognitive training. We applied a detailed questionnaire in order to exclude individuals that could cause biases of evaluation, without minimum score of 24 points in the Mini Mental State Examination and consciousness level compatible with the speech therapy evaluation. Then, the participants signed the Informed Consent Form. This study was approved by the Ethics Committee of the Gafrée Guinle University Hospital (HUGG) with the number 1.084.251, in accordance with the ethical standards inherent to researches involving human beings, considering Resolution 466 of 12 December 2012, of the Health National Council.

\section{- Experimental procedure}

Initially, we applied two cognitive evaluation questionnaires in order to evaluate the different aspects of short-term memory:

(1) Verbal fluency test (VFT) is a test of lexical

\section{TABLE 1: Patient profile.}

\begin{tabular}{|l|l|}
\hline Patient's Profile & \\
\hline Gender & Sample (\%) \\
\hline Male & $2(8.7)$ \\
\hline Female & $21(91.3)$ \\
\hline Age (years) & Mean \pm SD \\
\hline Minimum - Maximum: 63-91 & $76.4 \pm 7.4$ \\
\hline
\end{tabular}

access capability that provides information on the storage capacity of the semantic and spelling memory system, as well as the ability to retrieve information consolidated in memory.

(2) Mini Mental State Examination is a cognitive screening instrument aiming to exclude the elders that feature positive criteria for dementia.

The study occurred in three stages: Cognitive evaluation of the participants. Then, we developed the working memory training through DVD, lasting 1 hour, held in eight meetings for a week. Three months after completing the training, the revaluation was done in order to comply with the reliability of the Mini Mental State Examination.

\section{The working memory training program}

The DVD for working memory training was developed through 50 hours of image capture at a studio; eight hours of external images; 80 hours for art-completion of roughly 300 inserts of images with audio, among cards and pictures; in addition to 32 hours for audio completion. The final product consisted of three DVDs, with seven hours of stimuli, the first one containing a menu with three explanatory sessions, the second DVD, a menu with a first example of five practical memory training modules and a third DVD with a second example of five practical training modules, so that users could reinforce the training. Nevertheless, the training was applied only through DVD 1 and 2 .

The program uses memory training techniques available in the literature $[2,19]$. We started with a non-traditional approach to the training DVD, since it assumes the user engagement and potential improvement of cognitive functions. Given this, the use of instructions for implementation is an effective strategy to improve the elders' cognition during the execution of specific tasks [2,19]. The main objective is to raise the participants' awareness about the positive effects of memory training. The first non-traditional approach consists of exploring automatic components of cognitive function, which does not decline with age, as it relates to the ease of adherence to certain behaviors that favor the memory development [19]. The DVD application is clarified at that stage through three sessions: the process of memory formation, automatic and voluntary attention, as well as the most effective strategies to improve the memory. The description of 
this session promotes greater participants' adherence and commitment with the training that will be offered, as well as facilitates retaining information during training.

In the sequence, the visual stimuli associated with auditory stimuli were divided into five practical modules, which included memorization techniques based on traditional approaches. The stimuli were inserted in modules in increasing levels of amount and complexity [1-3]. The selection criteria of the stimuli based on working memory components [20-22]. The Visual stimuli through image detection and discrimination of figures tend to facilitate the selective attention, influence the parahippocampal gyrus activation and can improve the short-term information storage [23]. In addition, playful or common daily stimuli were privileged in order to promote greater motivation during the task [20].

Furthermore, the proposition of name mnemonics techniques was developed through filming with 108 people, chosen randomly, in order to promote a greater proximity with the reality and avoid repetition of names in the task. The technique consists of memorizing information, which makes a new connection between information to be stored and the memory already consolidated, relating information in order to chain them together logically. The selection criteria of words and tasks presented in the exercises were chosen without semantic or phonological similarity, since they are easier to memorize. Lexical selection took into account only the morphological aspect. Initially, monosyllabic or disyllabic words were offered and with gradual syllabic increase, since plysyllabic words can produce cortical activation a bit slower than those with fewer syllables [24]. Logical activities were proposed because they are related to the increased speed of information processing and improved memory performance $[1,2]$. The selected exercises of logical reasoning are easily solved, considering the great difficulty of elders with this type of task. The aim was to minimize possible frustration while developing such activities.

\section{Statistical Analysis}

The variables were analyzed statistically by the $\mathrm{R}$ commander through absolute and relative frequency as well as mean and standard deviation. For comparison of the variables of the study, the non-parametric paired test of Wilcoxon was used. The significance level was fixed at $\mathrm{p} \leq 0.05$.

\section{Results}

The population consisted of 42 elderly individuals, attending the Reborn GroupHUGG, 19 were excluded (13 were absent in more than $20 \%$ of the cognitive training, five did not reach the minimum score in the MMSE and one did not have the minimum age for the study). The final sample consisted of 23 participants: $21(91.3 \%)$ elderly women and two $(8.7 \%)$ elderly men. The age group of the study population was $76.4 \pm 7.40$ years, with variation between 63 and 91 years (TABLE 1).

In relation to previous memory training, 12 individuals had already been through some type of training (52.2\%), all for over six months. When analyzing the professional responsible for this training, eight people reported having done with psychologist (66.7\%), one with geriatrician $(8.3 \%)$ and three could not name the professional (25.0\%).

TABLE 2 presents the results of cognitive assessments carried out by individuals before and after training. The highest score for the MMSE assessment is 30 points and for the selfassessment of working memory, 100 points. In relation to the frequency, in the eight weeks of implementation of the program, five individuals attended 100\%, eight individuals attended $87.5 \%$ and 10 individuals were in $75 \%$ of the training.

It also presents the median of the results of

TABLE 2: Results and significance pre-intervention and post-intervention.

\begin{tabular}{|c|c|c|c|c|c|}
\hline \multicolumn{6}{|c|}{ Pre-intervention and Post-intervention Significance } \\
\hline \multirow[b]{2}{*}{ Cognitive evaluation } & \multicolumn{4}{|c|}{ Results } & \multirow[t]{2}{*}{ p-value } \\
\hline & $\begin{array}{c}\text { Mean } \pm \text { SD } \\
\text { Before }\end{array}$ & Median Before & $\begin{array}{c}\text { Mean } \pm \text { SD } \\
\text { After }\end{array}$ & Median After & \\
\hline MMSE & $26.4 \pm 1.79$ & 26 & $28.9 \pm 1.6$ & 29 & $<0.001^{*}$ \\
\hline Self-evaluation & $60.0 \pm 15.06$ & 65 & $69.8 \pm 15.95$ & 70 & $0.002^{*}$ \\
\hline VFT - Semantic category & $13.9 \pm 3.77$ & 13 & $15.5 \pm 4.21$ & 16 & $0.022^{*}$ \\
\hline VFT - Spelling Criterion & $17.6 \pm 6.28$ & 17 & $18.3 \pm 7.65$ & 17 & 0.332 \\
\hline VFT - Free Evocation & $34.7 \pm 10.15$ & 34 & $37.7 \pm 12.53$ & 37 & 0.222 \\
\hline
\end{tabular}


the evaluations made by the participants of the survey after the completion of memory training on DVD. There was significance in the MMSE ( $\mathrm{p}=0.001$ ) (FIGURE 1), self-evaluation $(\mathrm{p}=0.002)$ (FIGURE 2) and verbal fluency test in the semantic category $(\mathrm{p}=0.022)$ (FIGURE 3). There was no statistical significance in the result of verbal fluency tests spelling criterion and free evocation. FIGURES 1-3 display the outcome of cognitive evaluations before

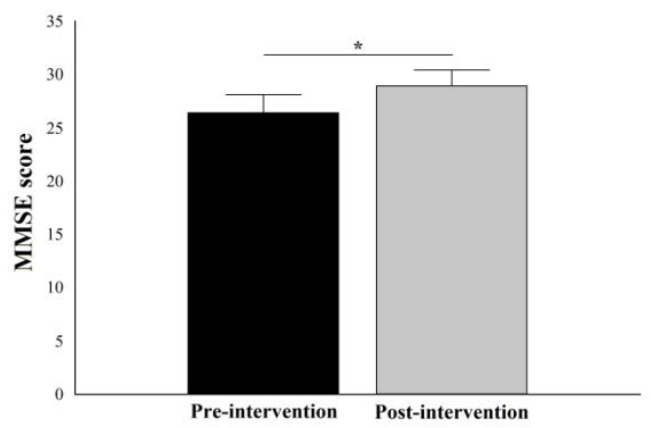

FIGURE 1: MMSE scores in pre- and postintervention conditions are presented by median + standard deviation. Significant results are highlighted with an asterisk (*).

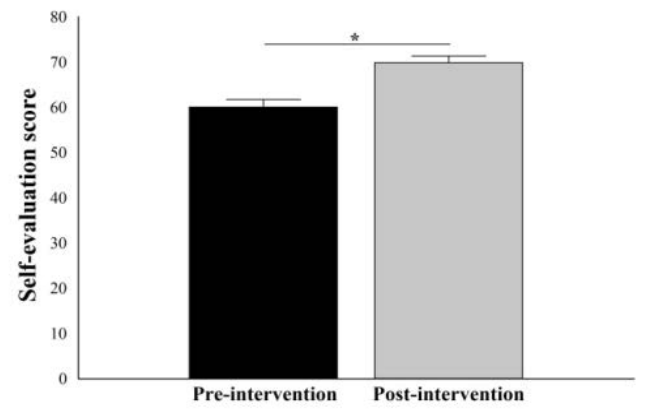

FIGURE 2: Self-evaluation scores in pre- and post-intervention conditions are presented by median + standard deviation. Significant results are highlighted with an asterisk (*).

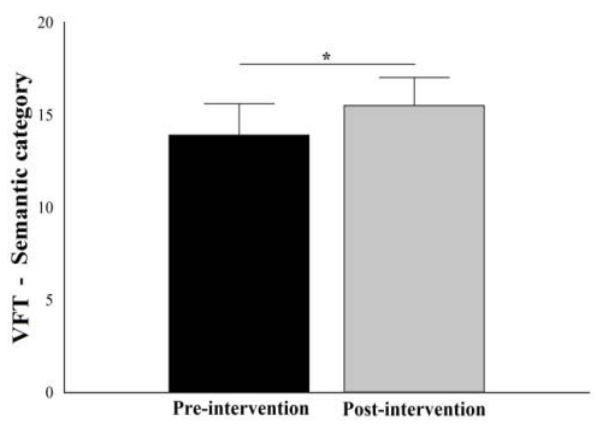

FIGURE 3: VFT - semantic category in pre- and post-intervention conditions are presented by median + standard deviation. Significant results are highlighted with an asterisk (*). and after the memory training conducted by individuals from the study.

\section{Discussion}

Cognitive training programs have hypothetically the potential to minimize agerelated memory decline, extending the cognitive competence $[1,4,14,20]$, due to demonstrating an easily applicable multimedia format and gathering different techniques that stimulate the memory.

The findings related to traditional training approach were the ones with the best results. This type of approach assumes an improvement in the neurobiological aspect of memory, because the presence of an instructor and specific activities of memory training present greater cognitive benefits to its participants, since it reaches a wider range of the population included in the training $[2,6]$. In order to answer this question, researches have been developed with non-traditional memory training approaches using guidelines and automatic components of cognitive function, in addition to proposing social, intelective and emotional engagement $[2,19,20]$. This type of training bases on the orientation of the subject on ways of acquiring memory, in addition to using components that warn at any moment, requesting for help regarding information while performing the task. This way to assist during training enhances cognitive resources in the performance of cognitive tasks [19,20]. However, although the results indicate the patients' improvement in the sets of memory assessments, long-term cognitive gains should be treated with recurrent training. Based on that kind of approach, the DVD also contained information on the stages of acquisition of long-term memory formation and practical exercises that can improve the cognitive memory resources. In addition to guidance on the importance of having social and intellective activities permanently.

The increased elderly population generates the need for developing techniques or strategies that stimulate cognitive function, through affordable resources, easily used by the public. Our training proposal implements all these addressed issues, in order to overcome the limitations of traditional training, as difficulties to accessibility and cost $[1,2,25]$. Accordingly, updates through multimedia and training software evolved to provide new treatment and rehabilitation capabilities with consequent benefits to patients, including computerized and 
online memory training. Therefore, our study allows a modern and easily applicable resource, as well as minimizes the lack of familiarity of the study group with multimedia resources through DVD, making the training program more effective $[1,2]$.

After applying non-traditional approaches, the training program included the memorization techniques used in memory training studies $[2,3,10]$. The evaluations and training were selfexplanatory and exemplified. In the experiment with the DVD, the elders' evaluation started with the Mini Mental State Examination prepared by Folstein et al. [26], creating a cognitive function screening, able to evaluate temporal and spatial orientation, short-term memory, evocation, attention and language skills. We can perceive the increased cognitive load with memory training $(\mathrm{p}<0.05)$. Analyzing this change, we can explain this increase primarily by the evocation item score (remembering three words, based on the mnemonic task), whose training participants began to feature better retention of information displayed for the visual stimuli tasks associated with auditory stimuli into five practical modules, which included the memorization techniques based on traditional approaches [26-28].

With respect to the self-evaluation outcome, the individuals' perception in relation to memory after training sessions improved, since the initial average was $65.0 \%$ and, on revaluation, it increased to $69.9 \%$. These improved answers, although discreet, represent the individuals' awareness regarding their cognitive abilities.

Moreover, the verbal fluency test showed that the organization of semantic information can be mediated by the training recurrence. Nevertheless, the different strategies used during the task, i.e. the individuals words production order, will lead various results. The efficiency in this test requires grouping words within subcategories, and changing for a new sub-category when the first one runs out. The groups rely on verbal memory processes and relate to the temporal lobe; on the other hand, the exchanges require mental and cognitive flexibility and relate to the frontal lobe and executive functions [15]. When we observe the findings in the study, the semantic category of the verbal fluency test showed significant difference between the mean of pre- and postmemory training results. Nonetheless, the other surveyed categories also showed an improved performance after cognitive training sessions.

The study presents some limitations, such as the sample size, heterogeneity regarding sex, and the absence of an electrophysiological variable that can measure the learning of new skills and maintenance of memory; however, it provides some support for understanding new methodologies of non-invasive memory training. We suggest larger sample and homogeneity of the target population in future studies in order to provide greater subsidies to improve the memory training instrument on DVD and achieve the goal of prolonging cognitive skills, with possibility of social integration and realization of daily functions. In addition to the concurrent analysis with electrophysiological mapping tools in cortical and subcortical areas involved in the neurobiological aspects of memory.

\section{Conclusion}

Implementing the training program of working memory on DVD can reduce the impact of the biological aspects of aging, in order to slow the age-related memory decline, from the inclusion of memory training techniques into daily life. Working memory training offers an alternative memory keeping, as well as treatment and rehabilitation in individuals with neurological disorders. Further advantages relate to a non-invasive and easily applicable treatment, which minimizes symptoms of memory deficits linked to neurological disorders. Working memory training through DVD differs from the existing treatments for being potentially simple, non-invasive, pharmacological and with low cost.

\section{Contributions}

The authors contributed equally.

\section{Conflict of interest}

The authors declare no potential conflict of interest. 
References

Ball K, Edwards JD, Ross LA. The Impact of Speed of Processing Training on Cognitive and Everyday Functions. J. Gerontol. B. Psychol. Sci. Soc. Sci. 62B, 1931 (2007).

Rebok GW, Michelle C, Carlson CM, Langbaum JBS. Training and maintaining memory abilities in healthy older adults: traditional and novel approaches $J$. Gerontol. B. Psychol. Sci. Soc. Sci. 62B, 5361 (2007).

Froudist-Walsh S, López-Barroso D, José Torres-Prioris M, Croxson P, Berthier ML. Plasticity in the Working Memory System: Life Span Changes and Response to Injury. Neuroscientist. 1, 1073858417717210 (2017).

Rolle CE, Anguera JA, Skinner SN, Voytek B, Gazzaley A. Enhancing Spatial Attention and Working Memory in Younger and Older Adults. J .Cogn. Neurosci. 29(9), 1483-1497 (2017)

Falbo S, Condello G, Capranica L, Forte R, Pesce C. Effects of PhysicalCognitive Dual Task Training on Executive Function and Gait Performance in Older Adults: A Randomized Controlled Trial. Biomed. Res. Int. 2016, 5812092.

Kelly ME, Loughrey D, Lawlor BA, Robertson IH, Walsh C, Brennan S. The impact of cognitive training and menal stimulation on cognitive and everyday functioning of healthy older adults: a systematic review and meta-analysis. Ageing Res. Rev. 15, 28-43 (2014).

Nielson JL, Paquette J, Liu AW, et al. Topological data analysis for discovery in preclinical spinal cord injury and traumatic brain injury. Nat.Commun. 6, 8581 (2015).

Fontes R, Ribeiro J, Gupta DS, et al. Time Perception Mechanisms at Central Nervous System. Neurol. Int. 8(1), 5939 (2016).

Park DC, Bischof GN. The aging mind: neuroplasticity in response to cognitive training. Dialogues Clin. Neurosci. 15(1), 109-119 (2013).
Baddeley A. Working Memory: looking back and looking forward. Nat. Rev. Neurosci. 4, 829-839 (2003).

Baddeley A, Hitch GJ. Development of working memory: should the PascualLeone and the Baddeley and Hitch models be merged? J. Exp. Child Psychol. 77(2), 128-137 (2000).

Cirulli ET, Kasperaviciūte D, Attix DK, et al. Common genetic variation and performance on standardized cognitive tests. Eur. J. Hum. Genet. 18(7), 815-820 (2010).

Montojo CA, Courtney SM. Differential neural activation for updating rule versus stimulus information in working memory. Neuron. 59(1), 173-182 (2008).

Hering A, Rendell PG, Rose NS, et al. Prospective memory training in older adults and its relevance for successful aging. Psychol. Res. 78(6), 892-904 (2014).

Teixeira S, Magalhães F, Marinho V, Velasques B, Ribeiro P. Proposal for using time estimation training for the treatment of Parkinson's disease. Med. Hypotheses. 95, 58-61 (2016).

Yesavage JA. Nonpharmacologic treatments for memory losses with normal aging. Am. J. Psychiatry. 142, 600-605 (1985).

Kliegl, RK, Smith, J, Baltes, PB. Testing the limits and the study of adult age differences in cognitive plasticity of a mnemonic skill. Dev. Psychol. 25(2), 247256 (1989).

Lachman ME, Weaver SL, Bandura ML, Elliott E, Lewkowicz CJ. Improving memory and control beliefs through cognitive restructuring and self-generated strategies. J. Gerontol. 47(5), 293-299 (1992).

Park DC, Gutchess AH, Meade ML, Stine-Morrow. EAL Improving Cognitive Function in Older Adults: Nontraditional Approaches. J. Gerontol. B. Psychol. Sci. Soc. Sci. 62B, 45-52 (2007).

Park JY, Jhung K, Lee J, An SK.
Theta-gamma coupling during a working memory task as compared to a simple vigilance task. Neurosci. Lett. 532(1), 3943 (2013).

Räma P. Domain-dependent activation during spatial and nonspatial auditory working memory. Cogn.Process. 9(1), 2934 (2008).

Alain C, Mcdonald KL, Kovacevic N, Mcintosh AR. Spatiotemporal Analysis of Auditory "What" and "Where" Working Memory. Cereb.Cortex. 19, 305-314 (2009).

Axmacher N, Schmitz DP, Wagner T, Elger CE, Fell J. Interactions between medial temporal lobe, prefrontal cortex, and inferior temporal regions during visual working memory. J. Neurosci. 28(29), 7304-7312 (2009).

Lewis G, Poeppel D. The role of visual representations during the lexical access of spoken words. Brain Lang. 134, 1-10 (2014).

Chan MY, Haber S, Drew LM, Park DC. Training older adults to use tablet computers: does it enhance cognitive function? Gerontologist. 56(3), 475-484 (2016).

Folstein MF, Folstein SE, McHugh PR. Mini-mental state: a practical method for grading the cognitive state of patients for the clinician. J. Psychiatric Res.12, 189198 (1975).

Krug RR, Silva AQAD, Schneider IJC, et al. Cognitive cooperation groups mediated by computers and internet present significant improvement of cognitive status in older adults with memory complaints: a controlled prospective study. Arq. Neuropsiquiatr. 75(4), 228-233 (2017).

Kavé G, Knafo-Noam A. Lifespan development of phonemic and semantic fluency: Universal increase, differential decrease. J. Clin. Exp. Neuropsychol. 37(7), 751-763 (2015). 